\title{
Óbito neonatal precoce e tardio: perfil das mães e recém-nascidos no estado de Rondônia
}

\author{
Early and late neonatal death: profile of mothers and newborns in the state of Rondônia
}

Muerte neonatal temprana y tardía: perfil de madres y recién nacidos en el estado de Rondônia

Larissa Nascimento Vilas Boas ${ }^{1 *}$, Charlie Darthanhã Lindenberg ${ }^{1}$, Daniel Ferreira Silva Cardoso Machado ${ }^{1}$, Gabriella Lucas Caetano ${ }^{1}$, Guilherme Rosa de Carvalho', Lucas Cabral Bellario', Safyra Zampieron Bezerra da Silva ${ }^{1}$, Thaís Henrique Matheus ${ }^{1}$, Sebastiana Linhares Pinto ${ }^{1}$, Yasmin Mendes Pinheiro'.

\section{RESUMO}

Objetivo: Identificar o perfil epidemiológico do binômio mãe-filho envolvidos em óbitos neonatais precoce e tardio em Rondônia. Métodos: Estudo quantitativo, exploratório e descritivo de série temporal, realizado através de consultas ao Sistema de Informação sobre Mortalidade (SIM). Elaborou-se um banco de dados acerca dos óbitos infantis no estado de Rondônia no período de 2014 a 2018, delimitando-se microrregiões e variáveis como local de parto. Resultados: No que tange a microrregião, observa-se que Porto Velho destaca-se com $39 \%$ em um total de 1.234 óbitos. Na variável faixa etária materna houve domínio do intervalo de 20 a 24 anos, contabilizando 392 óbitos. Na variável local de parto constatou-se que no âmbito intrahospitalar ocorreram 384 óbitos totalizando 97,95\%, enquanto extra-hospitalar ocorreram apenas 5 . Tipo de gestação, sexo do neonato e cor/raça também apresentaram altas porcentagens. As demais variáveis presentes no estudo não apresentaram discrepância percentual importante. Conclusão: Apesar de haver programas e projetos governamentais voltados a saúde da gestante e do neonato, constata-se que o número de óbitos neonatais ainda é um entrave à saúde pública, deste modo, observa-se uma necessidade de intensificação nas medidas de prevenção à óbitos do binômio mãe-filho no momento do parto e pós-parto.

Palavras-chave: Recém-nascido, Mortalidade, Perfil epidemiológico, Saúde pública.

\section{ABSTRACT}

Objective: To identify the epidemiological profile of the mother-child binomial involved in early and late neonatal deaths in Rondônia. Methods: Descriptive exploratory quantitative study of a time series, carried out through consultations with the Mortality Information System (SIM). A database was created on infant deaths in the state of Rondônia from 2014 to 2018, delimiting micro-regions and variables such as place of birth. Results: Regarding the microregion, it is observed that Porto Velho stands out with $31.76 \%$ of a total of 1234 deaths. There was dominance in the variables maternal age group in which in the range of 20 to 24 years there were a total of 392 deaths, in the variable place of birth it was found that within the hospital there were 384 deaths totaling $97.95 \%$, while outside the hospital there were only 5 , type of pregnancy and color/race also showed high percentages. The other variables present in the study did not display a significant percentage discrepancy. Conclusion: Although there are governmental programs and projects aimed at the health of pregnant women and newborns, it appears that the number of neonatal deaths is still an obstacle to public health. Thus, there is a need to intensify measures to prevent deaths in the mother-child binomial at the time of delivery and postpartum.

Keywords: Newborn, Mortality, Epidemiological profile, Public health.

\section{RESUMEN}

Objetivo: Identificar el perfil epidemiológico del binomio madre-hijo involucrado en las muertes neonatales tempranas y tardías en Rondônia. Métodos: Estudio cuantitativo, descriptivo, de series de tiempo, realizado mediante consultas al Sistema de Información de Mortalidad (SIM). Se creó una base de datos sobre muertes infantiles en el estado de Rondônia de 2014 a 2018, delimitando microrregiones y variables como el lugar de nacimiento. Resultados: En cuanto a la microrregión, se observa que Porto Velho se destaca con el 31,76\%

${ }^{1}$ Centro Universitário São Lucas (UNISL), Porto Velho - RO. *E-mail: larissavb1@hotmail.com 
de un total de 1234 defunciones. Hubo predominio en las variables grupo de edad materna en el cual en el rango de 20 a 24 años hubo un total de 392 defunciones, en la variable lugar de nacimiento se encontró que dentro del hospital hubo 384 defunciones totalizando 97,95\%, mientras que fuera el hospital solo hubo 5, el tipo de embarazo y el color / raza también mostraron altos porcentajes. Las otras variables presentes en el estudio no mostraron una discrepancia porcentual significativa. Conclusión: Si bien existen programas y proyectos gubernamentales dirigidos a la salud de la gestante y del recién nacido, parece que el número de muertes neonatales sigue siendo un obstáculo para la salud pública, por lo que es necesario intensificar las medidas para prevenir las muertes en el binomio madre-hijo en el momento del parto y el puerperio.

Palabras clave: Recién nacido, Mortalidad, Perfil epidemiológico, Salud pública.

\section{INTRODUÇÃO}

A condição de saúde de uma determinada população pode ser determinada pelos indicadores de saúde que se configuram a partir de dados originados da sociedade, dentro desse grupo de indicadores podemos citar a mortalidade infantil que é considerada um dos melhores parâmetros para analisar a qualidade de vida e bem-estar social de uma população, sendo mais sensível em descrever a realidade (PINHEIRO CP, et al., 2020; FARIA R, 2016).

Segundo estudos realizados em sete estados brasileiros e no Distrito Federal entre os anos 2010 e 2015, ocorreu uma estreita diminuição da mortalidade infantil. No entanto, as regiões Norte e Nordeste permanecem com as maiores taxas de mortalidade infantil, sendo que em 2010 a taxa de mortalidade neonatal da região Norte foi de 14,6, contrastado à taxa nacional de 11,2. Portanto, essa diminuição está diretamente relacionada ao conjunto de tecnologias em procedimentos mais eficazes e intervenções em saúde, que tem como objetivo reduzir os índices (MUTTI CF, et al., 2020)

Contudo, a mortalidade neonatal constitui-se como um sério entrave na saúde pública brasileira, uma vez que ainda é considerada alta, além disso se configurar como um marcador de qualidade do serviço de assistência neonatal que é capaz de evidenciar as dificuldades ainda presentes na atenção primária à saúde, que é um dos responsáveis pelo acompanhamento pré-natal das gestantes (FILHO ACAA, et al., 2017).

Mais de $70 \%$ dos óbitos neonatais poderiam ser evitados caso houvesse uma assistência primária relacionada aos cuidados da gestante e do recém-nascido com boa cobertura. A morte neonatal reflete nas condições de vida da população, e, por consequência, proporciona impactos na saúde pública. Portanto, a identificação do problema e aplicação de ações apropriadas podem resultar na redução dos óbitos neonatais (MENDONÇA SM, et al., 2018).

Nessa vertente, o Ministério da Saúde executou o Projeto de Apoio à Implementação da Rede Cegonha no Brasil, sendo realizado através do Sistema Único de Saúde (SUS) para intervir na situação problema em questão, com o objetivo de diminuir a mortalidade materna e infantil e assegurar uma gestação, pré-natal, parto e um pós-parto seguros para a progenitora e para o neonato, além de prestar atendimento e cuidados para a criança nos seus dois primeiros anos de vida (BARBOSA MM, et al., 2021).

Por definição, tem-se que a Mortalidade Neonatal (MN) é definida pelos óbitos ocorridos entre os 27 primeiros dias de vida, sendo ainda subdividida em precoce e tardia, em que os óbitos ocorridos até o $6^{\circ}$ dia são considerados precoces enquanto os ocorridos entre $07^{\circ}$ e $27^{\circ}$ dia são conceituados como tardios (ARAUJO FILHO ACA, et al., 2017).

A mortalidade neonatal é apontada como indicador de nível de vida e bem-estar social da população, uma vez que os óbitos na primeira semana de vida refletem as condições de assistência que a gestante e o neonato recebem. É necessário o atendimento de saúde com qualidade para acompanhar todo desenvolvimento gestacional até o nascimento do bebê, com êxito em todos os ciclos da gestação, isso favorece o prognóstico materno e redução de agravos, proporcionando a maior sobrevida dos recém-nascidos (BARROS SS, et al., 2019).

Segundo a Organização das Nações Unidas (ONU), há uma diferença significativa de óbitos neonatais quando se compara países não desenvolvidos com países desenvolvidos, uma vez que o último apresenta 
um índice de óbito neonatal entre três a seis vezes maior, exemplo disso é a Itália, que em 2017 apresentou um índice de 3 mortes para cada 1.000 nascidos vivos, uma das menores taxas do mundo, enquanto o Brasil apresentou uma taxa quatro vezes maior (SILVA ACFA, et al., 2019).

É importante ressaltar que cerca de $74,4 \%$ dos óbitos neonatais ocorrem na primeira semana de vida, de modo que, $41,2 \%$ dos óbitos ocorrem nas primeiras vinte e quatro horas de vida. Os principais fatores de risco envolvem a assistência prestada à gestante durante a gestação através do pré-natal, o sexo e o peso do neonato, malformações congênitas e a qualidade do amparo durante o trabalho de parto (GAIVA MAM, et al., 2020).

Além disso, acerca do perfil materno, o grupo de risco conta com a faixa etária da mãe entre 20 a 34 anos, associado a escolaridade e a nível socioeconômicos baixos, já para gestantes cima de 35 anos, constatouse problemáticas como asfixia intraparto, por fim, mães com comorbidades, como hipertensão, que levam o feto a algum mal súbito (SILVA VMC, et al., 2019).

O Coeficiente de Mortalidade Infantil (CMI), é responsável por estimar acerca do perigo que crianças nascidas vivas possam vir a óbito dentro dos seus primeiros meses de vida, refletindo, portanto, no desempenho que as ações políticas e seus governantes tenham tido mediante a esse grupo específico. Os óbitos infantis podem ser subdivididos em evitáveis ou reduzíveis, total ou parciais por ações efetivas acessíveis dos serviços de saúde referido a um lugar ou período determinado (PÍCOLI RP, et al., 2019).

O Brasil avançou sua taxa de CMI de forma significativa no período pós-neonatal (1990 a 2012), contudo, as políticas públicas ainda não tiveram eficácia de fato no que tange a atenção necessária que as mulheres precisam no período antes, durante e depois de sua gestação, de forma que gera um nível de mortalidade infantil precoce alto, com antecedentes fisiológicos precários como anomalias, prematuridade, baixo peso até ocorrências patológicas como doenças infecciosas e parasitárias. Em Porto Velho, capital do estado de Rondônia, a taxa de CMI dentro de quatro anos (2006 a 2010) teve um crescimento de $26 \%$ tendo como um dos pilares a falta de assistência no pré-natal, exemplificando a falha da gestão de saúde nesse quesito. (FREITAS JLG, et al., 2020).

Neste cenário, estudos mostraram empecilhos nos itinerários de mulheres com baixo grau de instrução escolar, mães que não possuem companheiro, gestantes de baixa idade (10 a 19 anos) e gestantes em faixa etária extremas, associado ao difícil acesso ao pré-natal. Estima-se que cerca de $70 \%$ dos óbitos neonatais são fatores evitáveis caso haja atendimento de qualidade no pré-natal (SILVA ACFA, et.al., 2019).

A exemplo disso, um estudo realizado no Piauí mostrou que de 2008 a 2017 registraram-se 8.088 óbitos neonatais, onde $70,33 \%$ poderiam ter sido evitáveis, tendo como fator atenuante a atenção à mulher gestante representando cerca de $46,7 \%$ destas fatalidades e em segundo lugar a atenção ao parto adequada à mulher. Para a Organização Mundial da Saúde (OMS), é inaceitável uma taxa de mortalidade infantil acima de 10/1000, considerando-se de suma importância a redução dessas causas de mortes evitáveis em todo território (COSTA REAR, et al., 2020).

Portanto, por se tratar de um entrave que gera impactos na saúde pública, faz-se necessário a abordagem deste tema que tem como objetivo traçar o perfil epidemiológico das mães e dos recém-nascidos envolvidos na questão de óbitos neonatais precoce e tardio nos últimos cinco anos no estado de Rondônia, com enfoque na capital Porto Velho tendo em vista, os números significativos de mortalidade neonatais apresentados.

\section{MÉTODOS}

Trata-se de um estudo quantitativo, cujo dados foram obtidos por meio de consulta à base de dados Sistema de Informação sobre Mortalidade (SIM) através da interface "TabNet", disponibilizados pelo Departamento de Informática do Sistema Único de Saúde (DATASUS), acessado em outubro de 2020.

Dessa forma, elaborou-se um banco de dados e a coleta foi feita através da seleção do grupo de mortalidade: óbitos infantis, com escolha da área de abrangência: Rondônia, no período de 2014 a 2018. Das seleções disponíveis, primeiramente foi delimitado "Microrregiões IBGE", Porto Velho, Guajará Mirim, 
Ariquemes, Ji-Paraná, Alvorada D’Oeste, Cacoal, Vilhena e Colorado Oeste, por seguinte foi analisado apenas os números da capital do estado. Das seleções disponíveis foram definidas: faixa etária ( 0 a 6 dias e 7 a 27 dias), idade da mãe, tipo de parto, local de ocorrência, sexo do neonato, grau de instrução da mãe, a cor/raça e o peso ao nascer.

Por se tratar de um estudo que não realizou abordagens diretas a indivíduos, utilizando apenas banco de dados de domínio público, não houve necessidade de submissão ao Comitê de Ética em Pesquisa.

\section{RESULTADOS}

Com o intuito de obter o número de óbitos neonatais em todo o estado, primeiramente foi retirado e tabulado os dados referentes as microrregiões segundo o IBGE, para que posteriormente fosse analisado os casos que ocorreram na capital do estado e que estão disponíveis no "TabNet" na plataforma do DATASUS, no período de 2014 a 2018 (Tabela 1).

Tabela 1 - Óbitos de neonatos por microrregião segundo o IBGE entre os anos de 2014-2018.

\begin{tabular}{lccccccc}
\hline MICRORREGIÃO IBGE & $\mathbf{2 0 1 4}$ & $\mathbf{2 0 1 5}$ & $\mathbf{2 0 1 6}$ & $\mathbf{2 0 1 7}$ & $\mathbf{2 0 1 8}$ & Total N & Total \% \\
\hline Porto Velho & 95 & 110 & 102 & 93 & 82 & 482 & $39 \%$ \\
Guajará-Mirim & 20 & 12 & 16 & 20 & 21 & 89 & $7,2 \%$ \\
Ariquemes & 23 & 27 & 35 & 21 & 23 & 129 & $10,4 \%$ \\
Ji-Paraná & 34 & 47 & 34 & 36 & 45 & 196 & $15,8 \%$ \\
Alvorada D'Oeste & 9 & 7 & 6 & 9 & 6 & 37 & $2,9 \%$ \\
Cacoal & 40 & 42 & 27 & 20 & 25 & 154 & $12,4 \%$ \\
Vilhena & 19 & 26 & 21 & 25 & 18 & 109 & $8,8 \%$ \\
Colorado Oeste & 13 & 5 & 5 & 6 & 9 & 38 & $3 \%$ \\
Total & 253 & 276 & 246 & 230 & 229 & 1234 & $100 \%$ \\
\hline
\end{tabular}

Fonte: Boas LNV, et al., 2020; dados extraídos do Sistema de Informações sobre MortalidadeSIM/DATASUS.

Entre o período de 2014 a 2018, o estado de Rondônia contabilizou um total de 1.234 óbitos neonatais. Ao analisar a microrregião onde encontra-se a capital Porto Velho, constata-se que ela é maior responsável por óbitos neonatais no estado, visto que registrou 482 óbitos nesse período, cerca de $39 \%$ do total.

Ainda nessa vertente, avaliando os óbitos de neonatos de acordo com a idade materna na capital do estado de Rondônia, foi registrado um alto índice em mães dentro do intervalo de 20 a 24 anos, contabilizando o total de 101 óbitos no período entre 2014 a 2018, correspondendo a aproximadamente 25,7\% das mortes de neonatos em Porto Velho, seguido da faixa etária de 15 a 19 anos com 72 óbitos, já o intervalo de idade de 45 a 49 anos representou apenas $0,25 \%$ do total com apenas 1 óbito nos mesmos 5 anos (Tabela 2). Ao avaliar-se o sexo do neonato, verifica-se um número de 224 óbitos masculinos e 172 femininos no mesmo período (Tabela 3).

Tabela 2 - Óbitos de neonatos de acordo com a idade materna em Porto Velho entre os anos de 2014-2018.

\begin{tabular}{lccccccc}
\hline Idade mãe & $\mathbf{2 0 1 4}$ & $\mathbf{2 0 1 5}$ & $\mathbf{2 0 1 6}$ & $\mathbf{2 0 1 7}$ & $\mathbf{2 0 1 8}$ & Total N & Total \% \\
\hline $\mathbf{1 0}$ a 14 anos & 2 & - & 4 & 2 & - & 8 & $2 \%$ \\
$\mathbf{1 5}$ a 19 anos & 10 & 18 & 17 & 13 & 14 & 72 & $18,30 \%$ \\
$\mathbf{2 0}$ a 24 anos & 21 & 19 & 24 & 20 & 17 & 101 & $25,70 \%$ \\
$\mathbf{2 5}$ a 29 anos & 14 & 19 & 18 & 14 & 11 & 76 & $19,30 \%$ \\
$\mathbf{3 0}$ a 34 anos & 8 & 16 & 6 & 12 & 12 & 54 & $13,70 \%$ \\
$\mathbf{3 5}$ a 39 anos & 2 & 7 & 4 & 8 & 6 & 27 & $6,80 \%$ \\
$\mathbf{4 0}$ a 44 anos & 4 & 4 & 3 & - & 1 & 12 & $3 \%$ \\
$\mathbf{4 5}$ a 49 anos & - & - & - & - & 1 & 1 & $0,25 \%$ \\
Idade ignorada & 12 & 9 & 15 & 3 & 2 & 41 & $10,45 \%$ \\
Total & 73 & 92 & 91 & 72 & 64 & 392 & $100 \%$ \\
\hline
\end{tabular}

Fonte: Boas LNV, et al., 2020; dados extraídos do Sistema de Informações sobre Mortalidade- SIM/DATASUS. 
Tabela 3 - Óbitos de neonatos de acordo com o sexo em Porto Velho entre os anos de 2014-2018.

\begin{tabular}{lccccccc}
\hline Sexo & $\mathbf{2 0 1 4}$ & $\mathbf{2 0 1 5}$ & $\mathbf{2 0 1 6}$ & $\mathbf{2 0 1 7}$ & $\mathbf{2 0 1 8}$ & Total N & Total \% \\
\hline Masculino & 48 & 52 & 41 & 35 & 48 & 224 & $55,2 \%$ \\
Feminino & 35 & 34 & 34 & 32 & 37 & 172 & $42,3 \%$ \\
Ignorado & 2 & 3 & 1 & 2 & 2 & 10 & $2,5 \%$ \\
Total & 85 & 89 & 76 & 69 & 87 & 406 & $100 \%$ \\
\hline
\end{tabular}

Fonte: Boas LNV, et al., 2020; dados extraídos do Sistema de Informações sobre MortalidadeSIM/DATASUS.

Quanto ao peso ao nascer constatou-se que $27,3 \%$ correspondem a 500 a $999 \mathrm{~g}, 24,5 \%$ aos que nasceram com 1500 a $2499 \mathrm{~g}$, quanto aos $1000 \mathrm{~g}$ a 1499 corresponde a $20,6 \%$ e as menores porcentagens correspondem a informação ignorada (1,3\%), $4000 \mathrm{~g}$ e mais $(2,3 \%)$ e a menos de $500 \mathrm{~g}(2,5 \%)$ (Tabela 4$)$.

Tabela 4 - Caracterização dos óbitos de 2014 a 2018.

\begin{tabular}{lcc}
\hline Peso ao nascer & N & $\%$ \\
\hline Menos de $500 \mathrm{~g}$ & 10 & $2,5 \%$ \\
500 a $999 \mathrm{~g}$ & 107 & $27,3 \%$ \\
1000 a $1499 \mathrm{~g}$ & 81 & $20,6 \%$ \\
1500 a $2499 \mathrm{~g}$ & 96 & $24,5 \%$ \\
2500 a $2999 \mathrm{~g}$ & 45 & $11,5 \%$ \\
3000 a $3999 \mathrm{~g}$ & 39 & $10 \%$ \\
$4000 \mathrm{~g}$ e mais & 9 & $2,3 \%$ \\
lgnorado & 5 & $1,3 \%$ \\
Total & 392 & $100 \%$ \\
\hline
\end{tabular}

Fonte: Boas LNV, et al., 2020; dados extraídos do Sistema de Informações sobre MortalidadeSIM/DATASUS.

Ainda acerca da capital do estado, ao analisar os tipos e partos, constatou-se que o parto vaginal foi responsável por $68,5 \%$ dos óbitos, pois dentro de um total de 406 , encontrou que 278 ocorreram de forma vaginal, enquanto 122 partos foram do tipo cesárea (Tabela 5).

Tabela 5 - Óbitos de neonatos de acordo com o tipo de parto em Porto Velho entre os anos 2014-2018.

\begin{tabular}{lccccccc}
\hline Tipo parto & $\mathbf{2 0 1 4}$ & $\mathbf{2 0 1 5}$ & $\mathbf{2 0 1 6}$ & $\mathbf{2 0 1 7}$ & $\mathbf{2 0 1 8}$ & Total N & Total \% \\
\hline Vaginal & 56 & 57 & 51 & 53 & 61 & 278 & $68,5 \%$ \\
Cesário & 27 & 31 & 24 & 14 & 26 & 122 & $30 \%$ \\
Ignorado & 2 & 1 & 1 & 2 & - & 6 & $1,5 \%$ \\
Total & 85 & 89 & 76 & 69 & 87 & 406 & $100 \%$ \\
\hline
\end{tabular}

Fonte: Boas LNV, et al., 2020; dados extraídos do Sistema de Informações sobre MortalidadeSIM/DATASUS.

Ao averiguar-se a ocorrência de óbitos por local, notou-se que a maioria ocorreu em âmbito hospitalar, pois apenas 19 ocorreram em ambiente extra hospitalar, sendo 9 em domicílio e 1 em via pública, enquanto 387 se passaram no interior do hospital, totalizando uma porcentagem de 95,3\% (Tabela 6). Acerca do tipo de gestação, tem-se que 371 óbitos ocorreram em situações de gestação única, enquanto 28 advieram de uma gestação dupla e apenas 1 óbito se enquadrou em uma gestação tripla (Tabela 6).

Ao ser ponderado a cor/raça de cada um dos neonatos que vieram a óbito em Porto Velho, observou-se que $100 \%$ dos dados foram ignorados. Quanto ao grau de escolaridade materno baseado em anos, tem-se uma predominância de aproximadamente 34,7\% com 199 óbitos em mães que possuíam de 8 a 11 anos de escolaridade, por seguinte tem-se um número de 149 óbitos em mães que possuíam entre 4 a 7 anos de 
escolaridade, cerca de $26 \%$ do total. Em contrapartida, mães que não possuíam nenhuma escolaridade apresentaram o menor índice, com o total de 10 óbitos, representando cerca de $0,2 \%$ do total, já entre as mães que possuíam de 1 a 3 anos de escolaridade, verificou-se 51 óbitos, o correspondente a cerca de 8,9\%. (Tabela 6).

Tabela 6 - Variáveis dos óbitos neonatais de acordo com o ano de ocorrência em Porto Velho entre 20142018.

\begin{tabular}{|c|c|c|c|c|c|c|c|}
\hline Variáveis & 2014 & 2015 & 2016 & 2017 & 2018 & Total $\mathbf{N}$ & Total \% \\
\hline \multicolumn{8}{|l|}{ LOCAL DE OCORRÊNCIA } \\
\hline Hospital & 75 & 87 & 74 & 67 & 84 & 387 & $95,3 \%$ \\
\hline $\begin{array}{l}\text { Outro estabelecimento de } \\
\text { saúde }\end{array}$ & 1 & 1 & - & - & - & 2 & $0,5 \%$ \\
\hline Domicílio & 2 & 1 & 2 & 1 & 3 & 9 & $2,2 \%$ \\
\hline Via pública & 1 & - & - & - & - & 1 & $0,25 \%$ \\
\hline Outros & 1 & - & - & - & - & 1 & $0,25 \%$ \\
\hline Ignorado & 5 & - & - & 1 & - & 6 & $1,5 \%$ \\
\hline Total & 85 & 89 & 76 & 69 & 87 & 406 & $100 \%$ \\
\hline \multicolumn{8}{|l|}{ TIPO DE GESTAÇÃO } \\
\hline Única & 80 & 82 & 66 & 65 & 78 & 371 & $91,4 \%$ \\
\hline Dupla & 2 & 7 & 9 & 2 & 8 & 28 & $6,9 \%$ \\
\hline Tripla e mais & 1 & - & - & - & - & 1 & $0,25 \%$ \\
\hline Ignorado & 2 & - & 1 & 2 & 1 & 6 & $1,5 \%$ \\
\hline Total & 85 & 89 & 76 & 69 & 87 & 406 & $100 \%$ \\
\hline \multicolumn{8}{|l|}{ ESCOLARIDADE DA MÃE } \\
\hline Nenhuma & 4 & 2 & 1 & 2 & 1 & 10 & $0,2 \%$ \\
\hline 1 a 3 anos & 7 & 18 & 11 & 8 & 7 & 51 & $8,9 \%$ \\
\hline 4 a 7 anos & 29 & 40 & 30 & 30 & 20 & 149 & $26 \%$ \\
\hline 8 a 11 anos & 37 & 40 & 42 & 43 & 37 & 199 & $34,7 \%$ \\
\hline 12 anos ou mais & 13 & 17 & 12 & 9 & 13 & 64 & $11,2 \%$ \\
\hline Ignorado & 32 & 21 & 13 & 15 & 19 & 100 & $17,5 \%$ \\
\hline Total & 122 & 138 & 109 & 107 & 97 & 573 & $100 \%$ \\
\hline
\end{tabular}

Fonte: Boas LNV, et al., 2020; dados extraídos do Sistema de Informações sobre MortalidadeSIM/DATASUS.

\section{DISCUSSÃO}

Constata-se que a maioria dos óbitos na capital de Porto Velho $(R O)$ ocorreu em recém-nascidos do sexo masculino, contabilizando 220 óbitos, enquanto o sexo feminino contabilizou 167 óbitos no mesmo período. A faixa etária materna entre 20 e 24 anos foi a que mais contabilizou óbitos. Acerca da escolaridade da mãe, aquelas que estudaram entre 8 a 11 anos se sobressaíram. Além disso, gestações únicas e intra-hospitalares registraram maiores taxas de óbito.

De acordo com o artigo apresentado está o estudo realizado em Goiânia (GO) por Tobias CS, et al. (2019), indicando que a idade materna que apresenta maior porcentagem de óbitos neonatais em Goiânia (GO) no ano de 2015 é concordante com os dados apresentados em Porto Velho - Rondônia, dado que, o intervalo de idade mais frequente na capital goiana também é de 20 a 24 anos, ocupando uma porcentagem de 19,3\%, enquanto a faixa etária de 45 a 49 anos ocupa uma pequena porcentagem de $0,3 \%$ do total, sendo que em Porto Velho não houve nenhuma morte registrada nessa faixa etária, no ano de 2018.

Outros pontos semelhantes do presente estudo com o estudo de Tobias CS, et al. (2019), são neonatos do sexo masculino com um índice maior de óbito, maior índice de óbitos intra-hospitalares quando comparado aos extra-hospitalares, maior índice de óbito em mães com gestações únicas e com escolaridade entre 8 e 11 anos. 
Já na macrorregião do Rio Grande do Norte, segundo Souza AMG, et al. (2014), registraram-se 159 óbitos neonatais dentro da faixa etária materna de 15 a 24 anos, enquanto em Porto Velho no mesmo ano apresentou-se apenas 31 óbitos dentro da mesma faixa etária.

Reforçando os dados obtidos nesse presente artigo, Aguiar KV, et al. (2021), através de um ensaio acerca de óbitos por sepse neonatal no estado da Bahia, realizado em 2021, indivíduos recém-nascidos, do sexo masculino, com baixo peso ao nascer, nascidos de gestação unifetal por parto vaginal foram os que registraram maior percentual de óbito neonatal. Acerca do perfil materno, esse mesmo estudo constatou que a faixa etária das mães com maior prevalência de óbitos neonatais são aquelas entre 20 e 24 anos e 15 a 19 anos, com escolaridade entre 8 a 11 anos de estudo.

Encontrou-se, em um estudo realizado em Minas Gerais, por Rocha MO, et al. (2021), maior mortalidade infantil por malformação congênita no sexo masculino, peso nascer maior que $2.500 \mathrm{~g}$, cor da pele branca, de gravidez unifetal e de parto cesárea. A maior parte das mães apresentavam escolaridade entre 8 a 11 anos de estudo, e, acerca da faixa etária materna, prevaleceu a idade entre 20 a 29 anos. Contudo, esse estudo foi semelhante ao estudo realizado em Porto Velho (RO), em 2020, em que o maior índice de óbitos neonatais foi relatado em recém-nascidos do sexo masculino, de cor de pele branca e gravidez unifetal e em mães com escolaridade entre 8 e 11 anos e com faixa etária entre 20 e 24 anos.

Ressalta-se, através de dados relatados em um estudo realizado por Sena MRD, et al. (2020), maior índice de mortalidade neonatal em hospitais no Baixo Amazonas em mães com idade entre 16-20 anos, diferindo da análise realizada em Porto Velho (RO), onde a faixa etária materna prevalente é entre 20 a 24 anos, entretanto, o estudo realizado no Baixo Amazonas, da mesma maneira que o de Porto Velho, notou maior índice de óbitos neonatais em gestações únicas e de parto vaginal. Quanto aos neonatos, houve prevalência do sexo masculino, com parto intra-hospitalar, assim como no estudo realizado em Porto Velho (RO).

Encontrou-se, em um outro estudo, realizado por Silva LSR, et al. (2020), no estado do Maranhão com dados semelhantes ao estudo realizado em Porto Velho (RO) em 2021, visto que, houve predomínio da idade materna entre 20 e 29 anos no estudo realizado no Maranhão, enquanto em Porto Velho (RO) a idade materna com maior índice de óbito neonatal foi entre 20 e 24. Para Silva LSR, et al. (2020), houve destaque das mães com escolaridade entre 8 a 11 anos, com gestações unifetais, neonatos do sexo masculino e parto vaginal de ocorrência intra-hospitalar, em concordância com a presente análise realizada na capital de Rondônia.

Ainda acerca do estudo realizado em um município do Maranhão, por Silva LSR, et al. (2020), com o objetivo de apurar a proporção de óbitos fetais de acordo com as variantes pertencente ao parto, notou-se um maior percentual em parto via vaginal $(64 \% ; n=73)$, análogo ao presente estudo elaborado na cidade de Porto velho (RO), onde constatou que $68,5 \%(n=278)$ óbitos ocorreram via vaginal. Evidenciou-se também na pesquisa apresentada do Maranhão, conforme a localidade da ocorrência, em que quase a totalidade de óbitos (92,1\%; $n=105)$ transcorreram dentro de hospitais, assim como foi observado em Porto Velho (RO) em que $95,3 \%$ ( $n=387)$ dos óbitos neonatais foram procedentes de hospitais.

Acerca do peso do neonato ao nascer, o presente estudo evidência maior índice de óbitos nos recémnascidos com peso entre 500 a 999 gramas (27\%), seguido por aqueles que pesavam entre 1500 a 2499 gramas (24\%) e, posteriormente, por aqueles que pesavam entre 1000 a 1499 gramas $(21 \%)$ ao nascer, o que assemelha-se ao estudo realizado por Gaíva MAM, et al. (2020), onde observou-se que o risco de óbito neonatal é 9 vezes maior nos RN com o peso no intervalo de 500 a 999 gramas e 5 vezes maior nos RN que pesavam entre 1.000 a 1.499 gramas quando comparados aos que pesavam entre 1.500 e 2.499 gramas.

Por fim, ainda em concordância com os dados obtidos por Gaíva MAM, et al. (2020), o local onde mais ocorre óbitos neonatais trata-se de hospitais, atingindo uma porcentagem de $99 \%$, assim como mostra os resultados expressos no presente estudo. Ademais, a pesquisa realizada por Gaíva MAM, et al. (2020) corrobora com a pesquisa realizada em Porto Velho-RO em outras vertentes, como, por exemplo, o tipo de gestação com maior número de óbitos, sendo a gravidez única a que apresenta o maior índice de óbitos em ambos. Além disso, as análises estão de acordo acerca de maior número de óbitos em recém-nascidos do sexo masculino quando comparados ao sexo feminino. Entretanto, quando se analisa a variável "tipo de 
parto", constata-se uma divergência, uma vez que no estudo de Porto Velho-RO observou mais mortes em casos de partos vaginais, porém, segundo o exposto por Gaíva MAM, et al. (2020), há uma maior taxa de óbitos via parto cesáreo.

\section{CONCLUSÃO}

Neste estudo, abordou-se o tema óbitos neonatal precoce e tardio e suas variáveis, contribuindo então para fomento da educação pré e pós-natal. Constatou-se então que apesar de haver programas e projetos governamentais voltados a saúde da gestante e do neonato, o número de óbitos neonatais ainda é considerável quando se analisa os dados coletados neste estudo, deste modo é observado uma necessidade de maior intervenção educacional nas escolas e nas unidades básicas de saúde, que aborde a importância do completo acompanhamento pré-natal para rastreio e prevenção de complicações e doenças. Ainda na vertente da intervenção, também é necessário reforçar as ações quanto a coleta de dados completas, uma vez que conforme os dados apresentados há um número elevado de informações tidos como ignorados.

\section{REFERÊNCIAS}

1. AGUIAR KV, et al. Aspectos epidemiológicos dos óbitos por sepse neonatal no Estado da Bahia. Revista Eletrônica Acervo Saúde, 2021; 13(6): e7630.

2. BARBOSA MM, et al. Rede cegonha: avanços e desafios da gestão do ambiente hospitalar. Revista Eletrônica Acevo Saúde. 2021; 13(3): e6486.

3. BARROS SS, et al. Ações para reduzir a taxa de mortalidade neonatal precoce. Anais do primeiro encontro multiprofissional em urgência e emergência pediátrica e neonatal. Revista Eletrônica Acervo Saúde. 2020 ; (1): e6343.

4. COSTA REAR, et al. Evolução da mortalidade infantil por causas evitáveis no estado do Piauí: 2008 a 2017 . Revista Eletrônica Acervo Saúde. 2020; 12(10): e4312.

5. FARIA R. Geografia da mortalidade infantil do Brasil: variações espaciais e desigualdades territoriais. Revista GeoUsp Espaço e Tempo. 2016; 20(3): 602-618.

6. FILHO ACAA, et al. Aspectos epidemiológicos da mortalidade neonatal em capital do nordeste do Brasil. Revista Cuidarte. 2017; 8(3): 1767-1776.

7. FREITAS JLG, et al. Mortalidade infantil em uma capital do norte do Brasil: estudo de série temporal. Revista Eletrônica Acervo Saúde. 2020; 12(11): e4981.

8. GAIVA MAM, et al. Fatores associados à mortalidade neonatal em recém-nascidos de baixo peso ao nascer. Revista Eletrônica Acervo Saúde. 2020; 12(11): e4831.

9. MENDONÇA SM, et al. Mortalidade neonatal no Brasil no período de 2004 a 2014. Revista Eletrônica Acervo Saúde. 2018; $11(2):$ e142.

10. MINISTÉRIO DA SAÚDE. DATASUS. Sistema de Informação sobre Mortalidade (SIM). Brasília. Brasil, 2020. Disponível em: http://www2.datasus.gov.br/DATASUS/index.php?area=02. Acesso em: 16 de outubro de 2020

11. MUTTI CF, et al. Caracterização de recém-nascidos internados em uma comunidade canguru da região norte do Brasil. Revista Eletrônica Acervo Saúde. 2020; (51): e3528.

12. PÍCOLI RP, et al. Mortalidade infantil e classificação de sua evitabilidade por cor ou raça em Mato Grosso do Sul. Ciência \& Saúde Coletiva. 2019; 24(9): 3315-3324.

13. PINHEIRO CP, et al. Produção cientifica acerca da mortalidade neonatal na primeira semana de vida. Revista Eletrônica Acervo Saúde. 2020; 20(12): e4900.

14. ROCHA MO, et al. Tendência temporal e perfil da mortalidade infantil por malformação congênita em uma região de saúde de Minas Gerais. Revista Eletrônica Acervo Saúde. 2021; 13(4): e6808.

15. SENA MRD, et al. Mortalidade neonatal em hospitais públicos de alta e média complexidade no Baixo Amazonas. Revista Eletrônica Acervo Saúde. 2020; (26): e2086.

16. SILVA ACFA, et. al. Índice de morte neonatal precoce: Uma análise do perfil materno. Revista Eletrônica Acervo Saúde. 2019; (26): e690.

17. SILVA LSR, et al. Perfil sociodemográfico e obstétrico dos óbitos fetais de gestantes residentes em um município do estado do Maranhão. Revista Eletrônica Acervo Saúde. 2020; (45): e3113.

18. SILVA VMC, et al. Fatores associados ao óbito fetal na gestação de alto risco: Assistência de enfermagem no prénatal. Revista Eletrônica Acervo Saúde. 2019; (37): e1884.

19. SOUZA AMG, et al. Perfil epidemiológico da mortalidade neonatal no Rio Grande do Norte - Brasil: Um estudo de base secundária. Revista ciência plural, 2018; 4(2):115-128.

20. TEIXEIRA GA, et al. Fatores de risco para a mortalidade neonatal na primeira semana de vida. Revista Cuidado é Fundamental Online, 2016; 8(1): 4036-4046.

21. TOBIAS GC, et al. Perfil dos óbitos infantis: um reflexo da assistência a saúde. Revista de enfermagem online, 2019; $13(4): 973-80$. 